\title{
Baja frecuencia de positividad serológica en pacientes con biopsias histológicamente compatibles con enfermedad celiaca en Perú
}

\author{
F. Arévalo ${ }^{1,2}$, E. Roe ${ }^{3}$, J. Arias-Stella Castillo ${ }^{1}$, J. Cárdenas ${ }^{4}$, P. Montes ${ }^{2,5}$ y E. Monge Can $^{2,5,6}$ \\ ${ }^{1}$ Instituto Anatomía Patológica y Biología Molecular Arias Stella ${ }^{2}$ Universidad Peruana de Ciencias Aplicadas. \\ ${ }^{3}$ Laboratorio Clínico ROE. ${ }^{4}$ Servicio Gastroenterología Clínica Internacional. ${ }^{5}$ Servicio Gastroenterología. Hospital \\ Daniel Alcides Carrión. ${ }^{6}$ Universidad Peruana Cayetano Heredia y Universidad Nacional Mayor de San Marcos. Perú
}

\section{RESUMEN}

Objetivo: estudiar la frecuencia de positividad de las pruebas serológicas en pacientes con biopsias compatible con enfermedad celiaca.

Material y métodos: estudio transversal. Se incluyeron pacientes con biopsia duodenal histológicamente compatible con enfermedad celiaca y determinación de anticuerpos antigliadina, antiendomisio y antitransglutaminasa IgA. Definimos como caso de enfermedad celiaca a quienes tuvieran biopsia positiva y anticuerpos antiendomisio y/o antitransglutaminasa positivos.

Resultados: 31 pacientes fueron incluidos de los cuales 6 fueron antiendomisio positivo, 5 fueron antitransglutaminasa positivo $y$ antigliadina fue positivo en 14 . Por lo tanto de 31 pacientes con cambios histológicos compatibles con enfermedad celiaca sólo 10 tuvieron serología diagnóstica. Sólo uno de los pacientes tuvo positividad tanto para antitransglutaminasa como para antiendomisio.

Conclusiones: a) encontramos que la mayoría de biopsias de duodeno con un cuadro histológico sugerente de enfermedad celiaca no se corresponden con serología diagnóstica de esta enfermedad; b) encontramos baja coincidencia en la positividad serológica entre antiendomisio y antitransglutaminasa.

Palabras clave: Enfermedad celiaca. Atrofia de vellosidades intestinales. Linfocitos intraepiteliales. Perú.

\begin{abstract}
Objective: to study the frequency of positive serology for celiac disease (CD) in patients with duodenal biopsies suggestive of this disease.
\end{abstract}

Material and methods: cross sectional study. We included patients with duodenal biopsies histologically compatible with $\mathrm{CD}$ and antigliadin, antiendomysial and IgA antitransglutaminase antibodies. We defined a "case" of CD if there was a positive biopsy and either antiendomisial or antitransglutaminase positive antibodies.

Results: thirty one patients were included in our study. Six were antiendomysial positive and 5 antitransglutaminase positive while the antigliadin was positive in 14 cases. Therefore, out of 31 patients only 10 had a serology compatible with $\mathrm{CD}$ and only one had positive both antibodies, antiendomysial and antitransglutaminase.

Conclusions: a) we have found that most of the duodenal biopsies compatible with $\mathrm{CD}$ are not diagnosed with positive serology; and b) we found a low correlation between serological diagnostic tests.

Key words: Celiac disease. Intestinal villous atrophy. Intraepithelial lymphocites. Perú.

Arévalo F, Roe E, Arias-Stella Castillo J, Cárdenas J, Montes $P$, Monge E. Baja frecuencia de positividad serológica en pacientes con biopsias histológicamente compatibles con enfermedad celiaca en Perú. Rev Esp Enferm Dig 2010; 102: 372-375.

\section{INTRODUCCIÓN}

La enfermedad celiaca (EC) se diagnostica mediante una combinación de cambios histológicos y pruebas serológicas en pacientes con o sin síntomas. Clásicamente se sospechaba de EC en presencia de diarrea crónica y malabsorción intestinal. Más recientemente esto ha cambiado y debe considerarse este diagnóstico también cuando se enfrenta a un paciente con dispepsia y anemia ferropénica.

La presencia de atrofia de vellosidades y la infiltración de linfocitos en el epitelio de superficie en una biopsia duodenal se ha convertido en el estándar histológico 
de la EC; mientras que la positividad de los anticuerpos antitransglutaminasa (ATGt) y antiendomisio (AEm) ha reemplazado a la antigliadina como método diagnóstico serológico actual (1).

Dada la poca disponibilidad de pruebas serológicas en nuestro país, se ha vuelto conducta habitual iniciar el diagnóstico con la biopsia intestinal y en muchas ocasiones el diagnóstico final se hace sólo con la biopsia y el cuadro clínico compatible.

Nuestro objetivo fue estudiar la frecuencia de la positividad de las pruebas serológicas para EC en pacientes con histopatología compatibles de EC en el Perú.

\section{MATERIAL Y MÉTODOS}

Diseño: estudio observacional de corte transversal.

Pacientes: se incluyeron pacientes referidos al Instituto de Patología Arias Stella que cumplieran los siguientes criterios:

- Pacientes adultos con presencia de síntomas gastrointestinales altos.

- Biopsias histológicamente compatibles con EC. Para este fin se incluyeron aquellas biopsias en la que el reporte patológico indicara tanto atrofia de vellosidades como infiltración de linfocitos intraepiteliales.

Los pacientes fueron incluidos desde febrero 2008 hasta mayo 2009. Todos los pacientes tuvieron una determinación de anticuerpos antigliadina, antiendomisio y antitransglutaminasa. Se hizo igualmente determinación de hemoglobina, vitamina B12 y ácido fólico. Se definió como anemia a valores de hemoglobina menores de 13 g/dl para varones y $11 \mathrm{~g} / \mathrm{dl}$ para mujeres, el déficit de ácido fólico fue definido como niveles menores de $3 \mathrm{ng} / \mathrm{ml}$ y el déficit de vitamina B12 como valores menores a 160 $\mathrm{pg} / \mathrm{ml}$.

Además, 14 de los 31 casos fueron sometidos a una prueba de aliento para descarte de sobrecrecimiento bacteriano intestinal alto.

Criterios de exclusión: se excluyeron aquellos pacientes con diagnóstico de neoplasias gastrointestinales, VIH asociado a enfermedad gastrointestinal, diagnóstico concomitante de enteroparásitos.

Biopsia compatible con EC: las biopsias duodenales fueron consideradas compatibles con enfermedad celiaca, si se encontraba incremento anormal de linfocitos intraepiteliales y atrofia de vellosidades, ambos definidos como:

-Incremento anormal de linfocitos intraepiteliales, definido como la presencia de 25 o más linfocitos por cada 100 células epiteliales $(2,3)$ al menos en 3 diferentes campos de $40 \mathrm{X}$.

- Atrofia de vellosidades definida como una relación de vellosidad/cripta menor de 1/3 en más de un campo de $10 \mathrm{X}$ en una muestra bien orientada (4).

Las biopsias duodenales fueron tomadas de la segunda porción duodenal. No consideramos a aquellos pacientes que teniendo incremento de linfocitos intraepiteliales no presentaran atrofia. La atrofia fue clasificada en leve, moderada y severa según la clasificación de Marsh (IIIa, IIIb y IIIc) (5).

Estudio serológico: el anticuerpo antitransglutaminasa IgA humano fue determinado usando una prueba de ELISA indirecta mediante el paquete comercial InmuLisa (IMMCO Diagnosis). El valor de corte utilizado fue de $20 \mathrm{U} / \mathrm{ml}$.

El anticuerpo antigliadina IgA se determinó usando una prueba ELISA indirecta con el mismo kit antes mencionado. Valor de corte fue de $20 \mathrm{U} / \mathrm{ml}$.

Los anticuerpos antiendomisio se determinaron utilizando esófago de mono como sustrato y una prueba de inmunofluorescencia indirecta (Scimex corporation). El valor de corte en este caso fue de 1:5 de dilución.

Los datos del estudio se procesaron utilizando el programa estadístico SPSS 15.0. Se utilizó la prueba t de Student para la comparación de promedios y la prueba exacta de Fisher para la comparación proporciones.

Definición de caso de enfermedad celiaca: se consideró como caso de EC a aquellos que cumpliendo los criterios de inclusión tuvieran positividad serológica a anticuerpo antiendomisio y/o anticuerpo antitransglutaminasa.

\section{RESULTADOS}

El presente estudio se realizó durante el periodo marzo 2008-abril 2009, durante el cual se incluyeron 31 pacientes que cumplieron con los criterios antes señalados.

De acuerdo a nuestra definición de caso, 10 pacientes $(32,3 \%)$ fueron considerados como casos de EC, la positividad de cada anticuerpo está expresada en la tabla I. Sólo 1 paciente presentó positividad para anticuerpos antitransglutaminasa y antiendomisio. Por otro lado $14 \mathrm{de}$ los 31 pacientes fueron negativos a todos los anticuerpos.

La tabla II muestra las características clínicas de los pacientes con enfermedad celiaca y de los pacientes con enteropatía no celiaca. No se encontraron diferencias entre ambos grupos.

En la tabla III mostramos la positividad de los 3 anticuerpos de acuerdo al grado de la atrofia intestinal. Se observa que la frecuencia de positividad de AEm y antigliadina se incrementan cuanto mayor es la atrofia. Esto no se observó con ATGt.

Tabla I. Positividad de la serología en pacientes con hallazgos histopatológicos compatibles con enfermedad celiaca

\begin{tabular}{lcc}
\hline & $n=31$ & $\%$ \\
\hline AGA & 14 & 45,16 \\
EMA & 6 & 19,2 \\
TTG & 5 & 16,1 \\
\hline
\end{tabular}


Tabla II. Características clínico-analíticas de acuerdo al diagnóstico final de enfermedad celiaca

\begin{tabular}{|c|c|c|c|}
\hline & $\begin{array}{l}\text { Enfermedad } \\
\text { celiaca }\end{array}$ & $\begin{array}{c}\text { Enteropatía } \\
\text { no celiaca }\end{array}$ & $p$ \\
\hline Edad años (rango) & $62,9 \pm 14,1(29-80)$ & $50,6 \pm 21,3(22-85)$ & 0,069 \\
\hline $\begin{array}{l}\text { Sexo } \\
\text { Mujeres } \\
\text { Hombres }\end{array}$ & $\begin{array}{l}8(80 \%) \\
2(20 \%)\end{array}$ & $\begin{array}{c}14(66,6 \%) \\
7(33,3 \%) \\
\end{array}$ & 0,67 \\
\hline Dispepsia & $10(100 \%)$ & 18 de $21(85,7 \%)$ & 0,53 \\
\hline Diarrea & $8(80 \%)$ & 19 de $21(90,4 \%)$ & 0,57 \\
\hline Anemia & 5 de $9(55,5 \%)$ & 6 de $19(31,5 \%)$ & 0,40 \\
\hline Déficit ácido fólico & 1 de $8(12,5 \%)$ & 5 de $17(29,4 \%)$ & 0,62 \\
\hline Déficit Vit. B12 & 4 de $10(40 \%)$ & 6 de $20(30 \%)$ & 0,69 \\
\hline
\end{tabular}

Prueba exacta de Fisher.

Tabla III. Positividad de los anticuerpos de acuerdo al grado de atrofia

\begin{tabular}{lccc}
\hline & Leve (MARSH I) & Moderada (MARSH II) & Severa (MARSH III) \\
\hline AGA $(\%)$ & $1(20)$ & $9(47,4)$ & $4(57,1)$ \\
\hline EMA $(\%)$ & $0(0)$ & $3(15,8)$ & $3(42,9)$ \\
\hline TTG & $0(0)$ & $5(26,3)$ & $0(0)$ \\
\hline Total & 5 & 19 & 7 \\
\hline
\end{tabular}

Se realizó la prueba de aliento de hidrógeno espirado para evaluar sobrecrecimiento bacteriano, encontrando sólo un caso positivo; este paciente además tenía una prueba serológica positiva para antiendomisio por lo que fue considerado como un caso de EC.

\section{DISCUSIÓN}

La EC es una entidad raramente diagnosticada en el Perú, por lo tanto existe poca información con respecto a la misma. Una búsqueda en las bases MEDLINE, LILACS y Scielo, mostró sólo un estudio realizado en el Perú (6). Una de las razones de este hecho es la poca disponibilidad de las pruebas serológicas de diagnóstico, razón por la cual, cuando se considera la posibilidad diagnóstica de EC en nuestro país, el diagnóstico se basa en la realización de biopsias duodenales. Debido a que son las biopsias lo que mayormente tenemos, nos propusimos como objetivo determinar con qué frecuencia se observa positividad en las pruebas serológicas en pacientes con una biopsia duodenal sugerente de EC. En el presente estudio sólo 10 de 31 casos $(32,3 \%)$ de biopsias compatibles fueron finalmente diagnosticados como EC. Estos resultados difieren de los de Ludwinson (7) y Giangreco (8) quienes han encontrado una alta positividad serológica en pacientes con atrofia vellositaria e incremento de linfocitos intraepiteliales (95 y 100\%, respectivamente).
Estas diferencias podrían ser explicadas porque en países en vías de desarrollo existen muchas otras causas de enteropatías de origen infeccioso que puedan dar similar patrón histológico. Nuestros resultados confirman que la evaluación histológica de atrofia vellositaria e infiltración linfocítica son inespecíficos y no deberían llevar de inmediato al diagnóstico de EC.

La deficiencia de IgA podría explicar bajas tasas de serología positiva en nuestros pacientes, sin embargo debemos notar que esta situación clínica es poco frecuente, tanto así que la búsqueda de esta deficiencia no es rutinaria.

También es de recordar que han sido descritos casos de EC con serología negativa, sin embargo estos suelen ser casos con daño histológico mínimo, algo que hemos tratado de descartar incluyendo en el presente reporte sólo pacientes con histología MARSH III (10).

Los AEm son considerados el gold-standard en el diagnóstico serológico de EC $(9,11)$, sin embargo la Asociación Gastroenterológica Americana (AGA) ha sugerido que sea la antitransglutaminasa la que se use como diagnóstico de EC en atención primaria (9). En nuestro estudio se ha encontrado una baja coincidencia en la positividad entre ambos anticuerpos. Seis pacientes fueron positivos para AEm, 5 para ATGt y sólo 1 de 31 presentó serología positiva para ambos. Esta falta de coincidencia ya ha sido observada por otros autores, habiendo varias posibles explicaciones. Una de ellas es el hecho que usan diferentes técnicas de laboratorio (inmunofluorescencia por un lado y ELISA por otro). Otra posibilidad es que pudiera haber otras proteínas diferentes a la transglutaminasa tisular actuando como sustrato para el antiendomisio $(10,12)$. Una tercera posibilidad es que se han encontrado estudios que usan 20 y hasta 2 $\mathrm{U} / \mathrm{ml}$ como nivel de corte (3).

Se ha descrito una predominancia del sexo femenino en la EC (13). En nuestro trabajo se encontró una relación F/M de 4.

La edad de presentación de la EC también ha sido de interés. Se sabe hoy que EC se puede ver en adultos tanto como en niños. Nuestro estudio incluyó sólo adultos y entre estos se puede ver que los pacientes varían desde el adulto joven hasta la tercera edad, con un promedio de edad por encima de los 60 años. Este dato es importante puesto que debe considerarse el diagnóstico de EC a toda edad.

La asociación entre grado de atrofia vellositaria y positividad serológica ha sido recientemente reportada, especialmente en población pediátrica (14-16). Nuestros resultados muestran que en el caso de AEm sí ocurre esta asociación y encontramos mayor positividad entre pacientes con mayor grado de atrofia. Sin embargo esto no se ha encontrado en el caso de ATGt. Nuestra serie es pequeña y estos resultados requieren de mayores estudios.

En nuestro estudio hemos encontrado un alto porcentaje de pacientes que teniendo una enteropatía con características histológicas de EC no han podido ser diagnostica- 
dos como tal. Existe una variedad de condiciones clínicas que pudieran explicar estos hallazgos histológicos, sprue tropical, intolerancia a proteínas dietarias y sobrecrecimiento bacteriano intestinal (SOBIA) entre ellas $(5,17)$. En 9 pacientes con serología negativa se realizó una prueba de aliento para SOBIA, siendo esta negativa en todos ellos.

Debemos reconocer que nuestros hallazgos ameritan confirmación con mayor número de pacientes, sin embargo nos permiten llegar a las siguientes conclusiones: a) la frecuencia con las que una biopsia duodenal compatible con EC se corresponde con serología diagnóstica es baja; y b) hemos encontrado una discrepancia en la positividad de los anticuerpos antiendomisio y antitransglutaminasa que nos llevan a sugerir que en nuestro país el diagnóstico de EC no debe basarse en una sola prueba serológica.

\section{BIBLIOGRAFÍA}

1. AGA Institute Medical Position Statement on the Diagnosis and Management of Celiac Disease. Gastroenterology 2006; 131: 1977-80.

2. Hayat M, Cairns A, Dixon MF, O'Mahony S. Quantitation of intraepithelial lymphocytes in human duodenum: what is normal? J Clin Pathol 2002; 55: 393-4.

3. Mariné M, Fernández-Bañares F, Alsina M, Farré C, Cortijo M, Santaolalla R, et al. Impact of mass screening for gluten -sensitive enteropathy in working population. World J Gastroenterol 2009; 15 : 1331-8.

4. Serra S, Jani PA. An approach to duodenal biopsies. J Clin Pathol 2006; 59: 1133-50

5. Antonioli D. Celiac disease: a progress report. Mod Pathol 2003; 16 : 342-6.
6. Perea VD, Fernan-Zegarra L, Cruz VM, Ballon R, Picoaga JL. Non specific ileoyeyunitis in Arequipa, Peru. Lancet 1978; 2: 550-2.

7. Ludvigson JF, Brandt L, Montgomery S, Granath F, Ekbon. A validation study of villous atrophy and small intestinal inflammation in Swedish biopsy registers. BMC Gastroenterol 2009; 9: 19.

8. Giangreco E, D’agate C, Barbera C, Puzzo L, Aprile G, Naso P, et al. Prevalence of celiac disease in adult patients with refractory functional dyspepsia. World J Gastroenterol 2008; 14: 6948-53.

9. Green P, Rostami K, Marsh M. Diagnosis of coeliac disease. Best Pract Clin Res Gastroenterol 2005; 19: 389-400.

10. American Gastroenterological Association (AGA) Institute Technical Review on the Diagnosis and Management of Celiac Disease. Gastroenterology 2006; 131: 1981-2002.

11. Chand N, Mihas AA. Celiac disease: current concepts in diagnosis and treatment. J Clin Gastroenterol 2006; 40: 3-14.

12. Utiyama SR, Nass FR, Kotze L, Nisihara RM, Ambrosio A, Messias IJ. Triagem sorológica de familiares de pacientes com doença celiaca: anticorpos anti-endomisio, anti-transglutaminase o ambos? Arq Gastroenterol 2007; 44: 156-61.

13. Megiorni F, Mora B, Bonamico M, Barbato M, Montuori M, Viola F, et al. HLA-DQ and susceptibility to celiac disease: evidence for gender differences and parent-of-origin effects. Am J Gastroenterol 2008; 103: 997-1003.

14. Fernández Salazar LI, de la Torre Ferrera N, Velayos Jiménez B, Nocito Colón M, González Hernández JM, Garrote Adrados JA. Problemas diagnósticos en la enfermedad celiaca del adulto. Rev Esp Enferm Dig 2008; 100: 24-8.

15. Ozgenc F, Aksu G, Aydogdu S, Akman S, Genel F, Kutukculer N, et al. Association between anti-endomysial antibody and total intestinal villous atrophy in children with coeliac disease. J Postgrad Med 2003; 49: 21

16. Donaldson MR, Firth SD, Wimpee H, Leiferman KM, Zone JJ, Horsley W, et al. Correlation of duodenal histology with tissue transglutaminase and endomysial antibody levels in pediatric celiac disease. Clin Gastroenterol Hepatol 2007; 5: 567-73.

17. Goldstein NS. Proximal small bowel intraepithelial lymphocytes. Histopathology 2004; 44: 199-205. 